\title{
DISPOSABLE IMMUNOASSAY CHIPS FOR HIV MONITORING IN RESOURCE-CONSTRAINED ENVIRONMENTS Chien-Fu Chen, ${ }^{1,2}$ Jikun Liu, ${ }^{1}$ Eric Wong, ${ }^{3}$ Chien-Cheng Chang, ${ }^{2}$ Indira Hewlett, ${ }^{3}$ Don L. DeVoe ${ }^{1 *}$ ${ }^{1}$ University of Maryland, USA \\ ${ }^{2}$ National Taiwan University, Taiwan \\ ${ }^{3}$ U.S. Food and Drug Administration, USA
}

\begin{abstract}
Here we describe the microfluidic components enabling disposable immunoassay chips for HIV diagnosis in resourceconstrained environments. The thermoplastic chip monitors the conserved viral capsid protein, p24 antigen. It combines blood sampling and plasma separation together with zero-power on-chip pumps and burst valves for manual flow control of sample and buffers. To enable sensitive p24 detection we have realized a polymer monolith porous support together with a novel anchoring chemistry allowing efficient covalent antibody immobilization. Using IgG as a model antigen to monolith-immobilized anti-IgG, an detection limit below $0.1 \mathrm{ng} / \mathrm{mL}$ has been achieved with an assay time of only $10 \mathrm{~min}$.
\end{abstract}

\section{INTRODUCTION}

Accurate, portable, and robust platforms for monitoring infectious diseases are critically needed to improve the quality of life for millions of people living in high-risk areas around the world. In particular, The HIV pandemic continues to be a significant worldwide public-health problem. The HIV virus is highly infective, rapidly mutates, and has no cure. Early diagnosis is critical for effective management. While most diagnostic assays detect HIV antibodies, the time for a host to produce these antibodies is up to six months after exposure, and newborns under 18 months of age cannot be tested with these methods due to masking by maternal antibodies. As an alternative, PCR-based nucleic acid testing (NAT) is highly sensitive, but labor-intensive and not suitable for resource-poor settings where simple, rapid, and inexpensive assays are required. Diagnostic tests targeting host antibodies or viral nucleic acids are also susceptible to false or discordant results due to viral variations.

Rather than detecting host antibody response, the thermoplastic chip described here is designed to directly monitor the conserved viral capsid protein, p24 antigen. While p24 detection provides exceptional specificity for monitoring early-stage infection, existing assays are not appropriate for use in resource-constrained environments where infrastructure, environmental, personnel, and financial resources can be highly limited [1]. To overcome these challenges we are developing a thermoplastic chip that combines blood sampling and plasma separation together with zero-power on-chip pumps and burst valves for manual flow control of sample and buffers. These functionalities are made possible by low-cost surface modifications based on UV-ozone exposure, enabling simple lithographic patterning of "microgap" channels and control over bond strength across different regions of the chip. To enable sensitive p24 detection we have realized a polymer monolith porous support together with a novel anchoring chemistry allowing efficient covalent antibody immobilization. The monolith sensors are shown to offer higher sensitivity than open-tubular flowthrough sensors, with significantly shorter assay times. The chip also provides for on-chip reagent and buffer storage, and safe storage of assay waste.

\section{RESULTS AND DISCUSSION}

A schematic of an integrated immunoassay chip is shown in Fig. 1. Whole blood from a finger prick is introduced into an inlet chamber, which is capped using a screw threaded into the thermoplastic substrate. The screw is manually actuated to flow $\sim 50 \mu \mathrm{L}$ of whole blood past a plasma extraction element to prevent fouling of the downstream detection elements by red blood cells. Manual pumps are also used to deliver rinse buffers and immunoassay reagents within the chip.

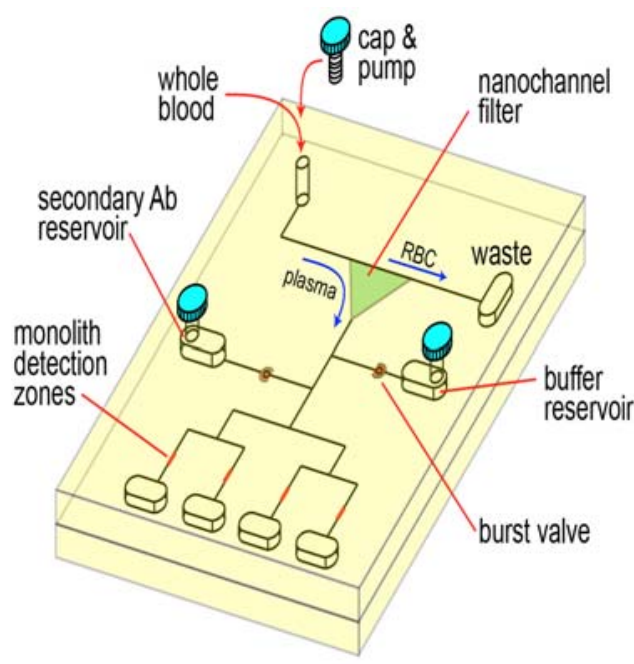

Figure 1: Schematic of the disposable thermoplastic HIV immunoassay chip.

A schematic of a manual screw pump is shown in Fig. 2, together with burst valves used to seal reagents on-chip. The valves are fabricated by controlling the thermoplastic bond strength through UV-ozone surface modifications.

A similar technique is used to fabricate the microgap plasma extraction element shown in Fig. 3. Details of this novel method for bond strength control and micro/nanochannel fabrication will be fully described. Detection of viral protein is achieved using antibodies immobilized on an array of porous epoxide monolith segments modified using a heterobifunctional crosslinking reagent (Fig. 4), ensuring high bioavailability of the antibodies. Detection may be performed using a direct or sandwich (Fig. 5) immunoassay format. Using IgG as a model antigen to monolithimmobilized anti-IgG in a direct assay, an excellent detection limit well below $0.1 \mathrm{ng} / \mathrm{mL}$ has been achieved with an assay time of only $10 \mathrm{~min}$ in a fully-manual format (Fig. 6). 

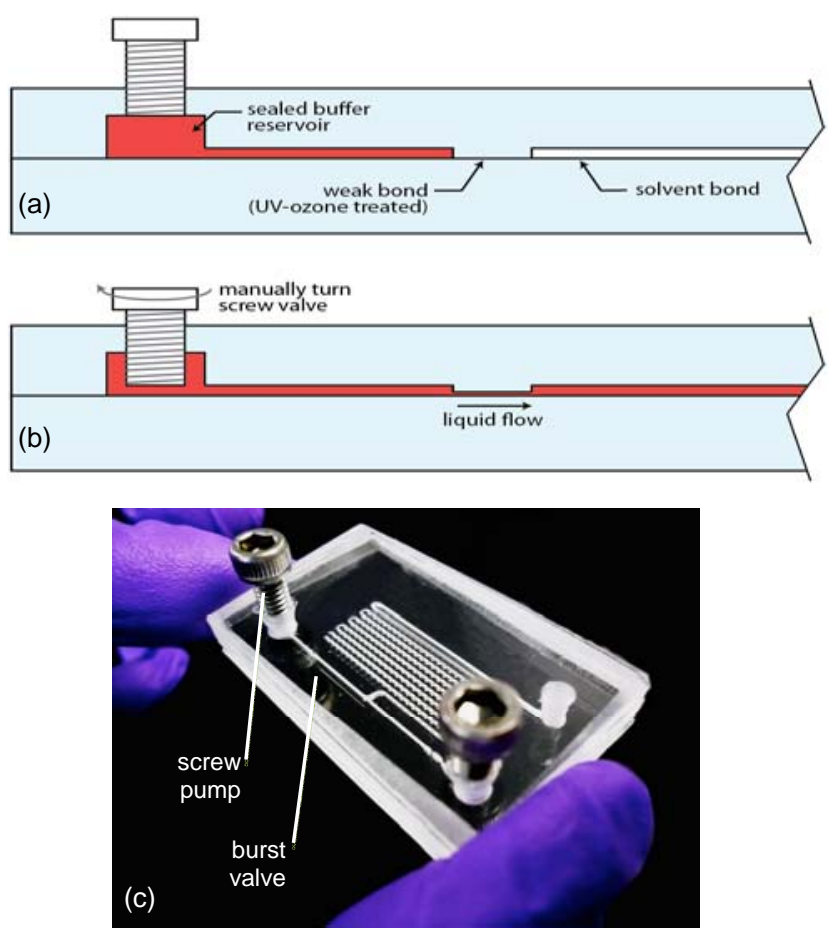

Figure 2: Buffer packet with integrated screw pump and burst valve (a) before and (b) after valve actuation, and (c) fabricated chip with 2 pumps and valves (actuated valve shown inset).
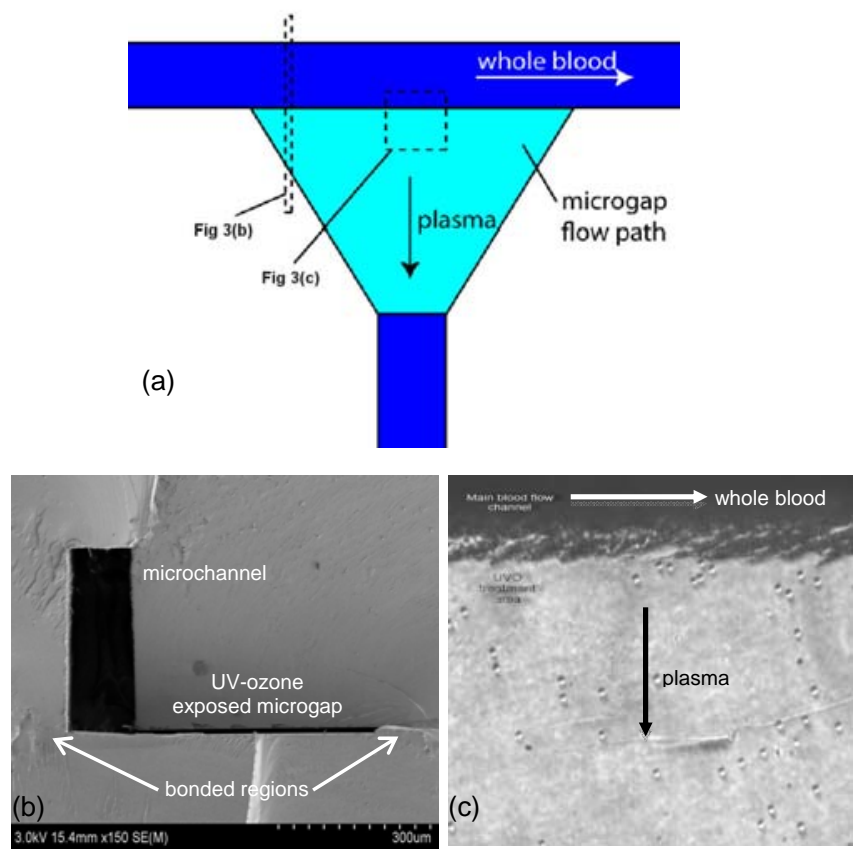

Figure 3: (a) schematic of a microgap plasma extraction element, (b) cross-sectional SEM image showing the sample injection microchannel seamlessly interfaced to a $\sim 4 \mathrm{~m}$ deep microgap fabricated by UV-ozone exposure and solvent bonding, and (c) isolation of plasma from whole blood using the microgap filter, with extraction efficiency above 95\% achieved in this example. The regions depicted in $(b-c)$ are noted in $(a)$.
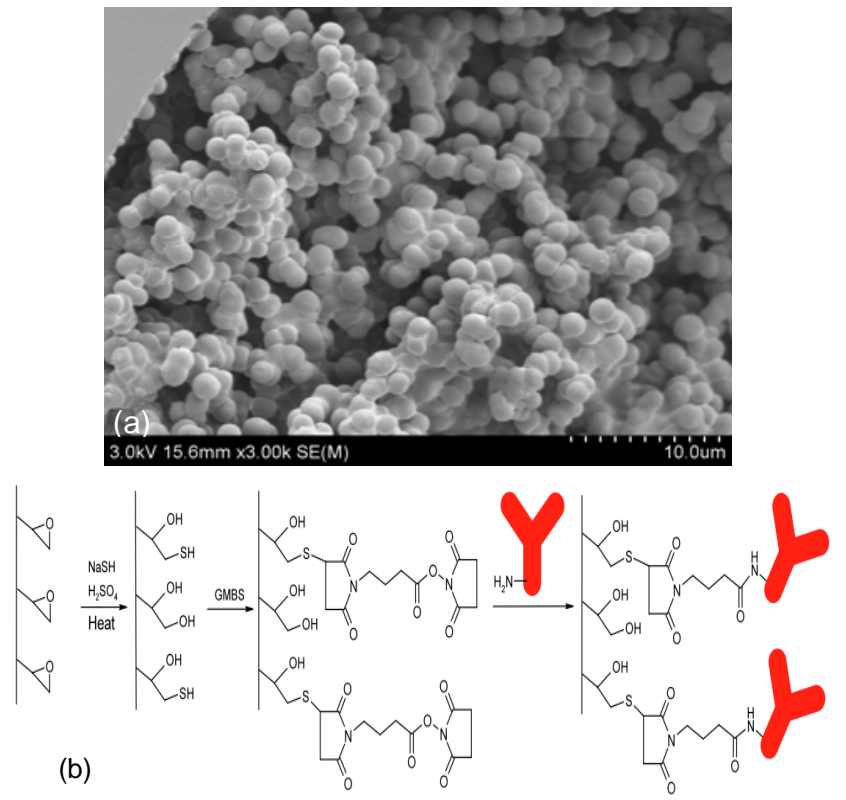

Figure 4: (a) SEM image of a porous monolith, and (b) antibody immobilization process using a heterobifunctional crosslinker.
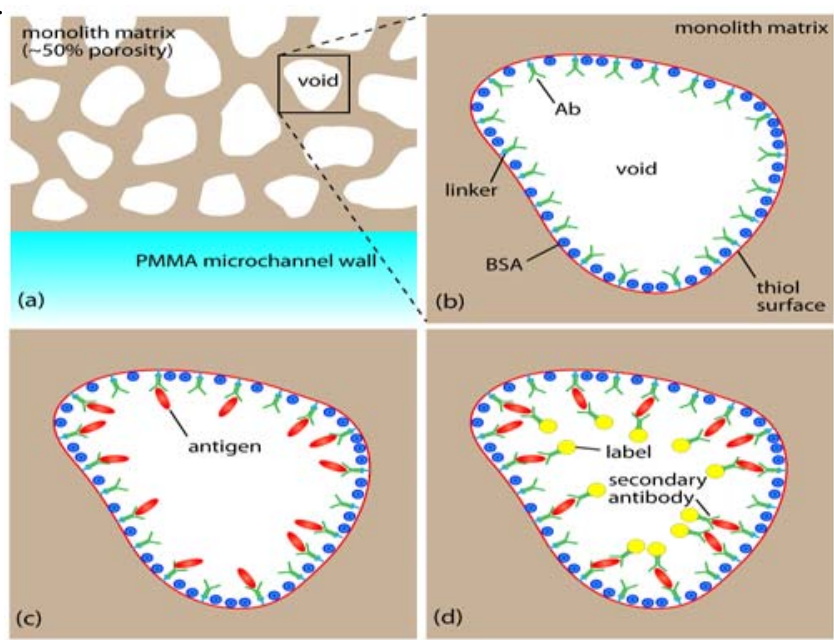

Figure 5: Sandwich assay steps performed within the porous monolith support matrix.

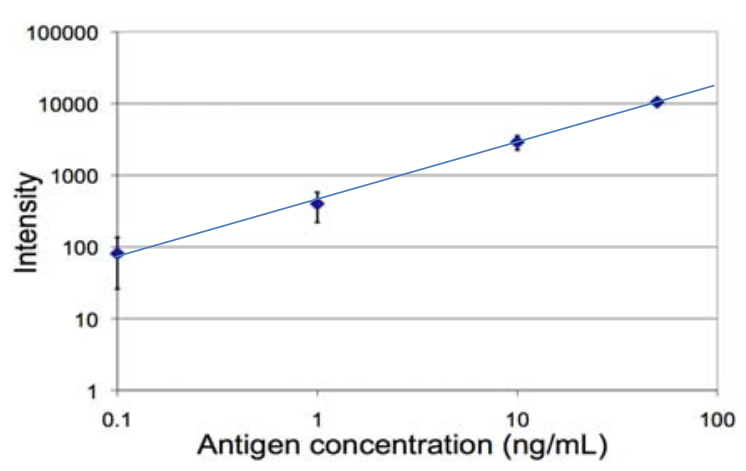

Figure 6: Dilution curve for rabbit IgG as a model antigen to monolith-immobilized anti-rabbit IgG. A limit of detection below $0.1 \mathrm{ng} / \mathrm{mL}$ is observed. 


\section{REFERENCES}

[1] B. Ledergerber, M. Flepp, J. Boni, Z. Tomasik, R. W. Cone, R. Luthy and J. Schupbach, "Human immunodeficiency virus type 1 p24 concentration measured by boosted ELISA of heat-denatured plasma correlates with decline in CD4 cells, progression to AIDS, and survival: comparison with viral RNA measurement". The Journal of Infectious Diseases. 181, 1280 (2000).

\section{CONTACT}

*Don L. DeVoe, tel: +1-301-405-8125; ddev@umd.edu 